\title{
Solving nonlinear programming problems with unbounded non-convex constraint sets via a globally convergent algorithm
}

Menglong Su${ }^{1 *}$, Jianhua $\mathrm{Li}^{1}$ and Zhonghai $\mathrm{Xu}^{2}$

"Correspondence:

mlsulynu@163.com

'school of Mathematical Sciences,

Luoyang Normal University,

Luoyang, 471022, P.R. China

Full list of author information is

available at the end of the article

\begin{abstract}
In this paper, we propose a set of unbounded conditions, under which we are able to solve nonlinear programming problems in a class of unbounded non-convex sets via the combined homotopy interior point algorithm. We also obtain the global convergence of the combined homotopy interior point algorithm and analyze the implementation of this algorithm in detail.
\end{abstract}

Keywords: unbounded conditions; nonlinear programming problems; non-convex sets

\section{Introduction}

Consider the constrained minimization problem:

$$
\begin{aligned}
\min _{x \in R^{n}} f(x) & \\
\text { s.t. } & u_{i}(x) \leq 0, \quad i=1, \ldots, m, \\
v_{j}(x) & =0, \quad j=1, \ldots, l,
\end{aligned}
$$

where $f: R^{n} \rightarrow R^{1}, u_{i}: R^{n} \rightarrow R^{1}, i=1, \ldots, m$ and $v_{j}: R^{n} \rightarrow R^{1}, j=1, \ldots, l$ are three times continuously differentiable.

We call a point $x^{*}$ is a Karush-Kuhn-Tucker (K-K-T) point of (1) and $y^{*}, z^{*}$ are the corresponding Lagrangian multiplier vectors if $\left(x^{*}, y^{*}, z^{*}\right)$ satisfies

$$
\begin{aligned}
& \nabla f(x)+\nabla u(x) y+\nabla v(x) z=0, \\
& v(x)=0, \\
& Y u(x)=0, \quad u(x) \leq 0, \quad y \geq 0,
\end{aligned}
$$

where $y \in R^{m}, z \in R^{l}, \nabla f(x)=(\partial f(x) / \partial x)^{T} \in R^{n}, \nabla u(x)=\left(\nabla u_{1}(x), \ldots, \nabla u_{m}(x)\right) \in R^{n \times m}$, $\nabla v(x)=\left(\nabla v_{1}(x), \ldots, \nabla v_{l}(x)\right) \in R^{n \times l}, u(x)=\left(u_{1}(x), \ldots, u_{m}(x)\right)^{T} \in R^{m}, v(x)=\left(v_{1}(x), \ldots\right.$, $\left.v_{l}(x)\right)^{T} \in R^{l}$ and $Y=\operatorname{diag}(y) \in R^{m \times m}$.

Since Kellogg et al. (see [1]) and Smale (see [2]) proposed the notable homotopy method, this method has become a powerful tool in dealing with various nonlinear problems, for 
example, zeros or fixed points of maps (see [3-5], etc. and the references therein). However, the homotopy method has seldom been touched upon in constrained optimization until 1988, Megiddo (see [6]) and Kojima et al. (see [7]) discovered the Karmarkar interior point method for linear programming was a kind of path-following method. From then on, the central path-following methods for mathematical programming have become an active research subject. Furthermore, it was extended to convex nonlinear programming problems recently (see [8-11], etc.). But all their convergence results were obtained under the assumptions that the logarithmic barrier function is strictly convex and the solution set is nonempty and bounded.

Recently, a combined homotopy interior point method (denoted as CHIP method for convenience) for nonlinear programming problems was presented in $[12,13]$ (detailed abstract of them was announced in [14]). In [13], compared with the central path-following methods, the authors removed the convexity of the logarithmic barrier function and the boundedness and nonemptiness of the solution set. This shows that the CHIP method can solve the problems that interior path-following methods cannot solve. In [15], by taking a piecewise technique, under the commonly used conditions, the polynomiality of the CHIP method was given, which shows that the efficiency of the CHIP method is also very well. In [16], we introduced $C^{2}$ functions $\alpha_{i}(x) \in R^{n}, i=1, \ldots, m$ and $\beta_{j}(x) \in R^{n}, j=1, \ldots, l$ to extend the results in $[12,17]$ to more general non-convex sets. However, there are no results reported in the literature about the work in [16] extended to unbounded non-convex sets. In this paper, we attempt to complete this work. To this end, by using some inequality techniques and the ideas of infinite solutions which were introduced in $[18,19]$, we develop a set of new unboundedness conditions. Under these conditions, we obtain the global convergence results of the CHIP method and therefore extend the work in [16] to unbounded non-convex sets.

\section{Main results}

In this section, the nonnegative and positive orthants of $R^{m}$ are denoted as $R_{+}^{m}$ and $R_{++}^{m}$, respectively. We also denote by $B(x)=\left\{i \in\{1, \ldots, m\}: u_{i}(x)=0\right\}$ the active set at $x$. In addition, let $X=\left\{x \in R^{n}: u_{i}(x) \leq 0, i=1, \ldots, m, v_{j}(x)=0, j=1, \ldots, l\right\}$ be the feasible set, let $X^{0}=\left\{x \in R^{n}: u_{i}(x)<0, i=1, \ldots, m, v_{j}(x)=0, j=1, \ldots, l\right\}$ be the strictly feasible set and let $\partial X=X \backslash X^{0}$ be the boundary set of $X$.

In [16], to solve (2), the following homotopy was constructed:

$$
\begin{aligned}
& H\left(w, w^{(0)}, \lambda\right) \\
& =\left(\begin{array}{c}
(1-\lambda)\left[\nabla f(x)+\nabla u(x) y+\lambda \xi(x) y^{2}\right]+[\nabla v(x)+\lambda(\eta(x)-\nabla v(x))] z+\lambda\left(x-x^{(0)}\right) \\
v(x) \\
Y u(x)-\lambda Y^{(0)} u\left(x^{(0)}\right)
\end{array}\right) \\
& =0,
\end{aligned}
$$

where $w=(x, y, z) \in R^{n+m+l}, w^{(0)}=\left(x^{(0)}, y^{(0)}, 0\right) \in X^{0} \times R_{++}^{m} \times R^{l}$, and $Y^{(0)}=\operatorname{diag}\left(y^{(0)}\right) \in$ $R^{m \times m}$.

In this section, we utilize the concept of infinite solutions $[18,19]$ and hence give a new set of unboundedness conditions. The nonlinear programming problem is said to have a solution at infinite, if there exists a sequence $\left\{x^{(k)}\right\}$ satisfying $\left\{x^{(k)}\right\} \subset X,\left\|x^{(k)}\right\| \rightarrow \infty$ as 
$k \rightarrow \infty$, and for any given $\zeta \in X$, there exist $y^{(k)} \in R_{+}^{m}$ and $z^{(k)} \in R^{l}$ such that

$$
\lim _{k \rightarrow \infty}\left(\zeta-x^{(k)}\right)^{T}\left(\nabla f\left(x^{(k)}\right)+\nabla u\left(x^{(k)}\right) y^{(k)}+\nabla v\left(x^{(k)}\right) z^{(k)}\right) \geq 0
$$

Then we assume that there exist smooth mappings $\xi_{i}(x) \in R^{1}, i=1, \ldots, m$ and $\eta_{j}(x) \in R^{1}$, $j=1, \ldots, l$ such that:

$\left(\mathrm{A}_{1}\right) X^{0}$ is nonempty; nonlinear programming problems have no infinite solutions.

$\left(\mathrm{A}_{2}\right) \forall x \in X$, if

$$
\sum_{i \in B(x)}\left(y_{i} \nabla g_{i}(x)+\alpha_{i} \xi_{i}(x)\right)+\nabla v(x) z+\eta(x) \beta=0, \quad y_{i} \geq 0, \alpha_{i} \geq 0, z \in R^{l}, \beta \in R^{l},
$$

then $y_{i}=0, \alpha_{i}=0$ and $z=\beta=0, \forall i \in B(x)$.

$\left(\mathrm{A}_{3}\right) \forall x \in X$, we have

$$
\left\{x+\sum_{i \in B(x)} y_{i} \xi_{i}(x)+z \eta(x): y_{i} \geq 0, i \in B(x), z \in R^{l}\right\} \cap X=\{x\}
$$

where $\eta(x)=\left(\eta_{1}(x), \ldots, \eta_{l}(x)\right)$.

$\left(\mathrm{A}_{4}\right) \forall x \in X, \nabla v(x)$ is of full of column rank and $\nabla v(x)^{T} \eta(x)$ is nonsingular.

However, only applying the infinite solution technique to the items $\nabla f(x), \nabla u(x)$, and $\nabla v(x)$, we cannot extend the results in [16] to unbounded cases because of the existence of the items $\xi(x)$ and $\eta(x)$. So for the items $\xi(x)$ and $\eta(x)$, we need to use other techniques. In this paper, we still need the following assumption:

$\left(\mathrm{A}_{5}\right) \forall \zeta \in X$

$$
u_{i}(\zeta)^{T}-u_{i}(x)^{T} \geq(\zeta-x)^{T} \xi_{i}(x) y_{i}, \quad i=1, \ldots, m, \quad v(\zeta)^{T}-v(x)^{T} \geq(\zeta-x)^{T} \eta(x)
$$

For a given $w^{(0)}$, rewrite $H\left(w, w^{(0)}, \lambda\right)$ as $H_{w^{(0)}}(w, \lambda)$. The zero-point set of $H_{w^{(0)}}$ is

$$
H_{w^{(0)}}^{-1}(0)=\left\{(w, \lambda) \in X \times R_{+}^{m} \times R^{l} \times(0,1]: H_{w^{(0)}}(w, \lambda)=0\right\} .
$$

The inverse image theorem tells us that, if 0 is a regular value of the map $H_{w^{(0)}}$, then $H_{w^{(0)}}^{-1}(0)$ consists of some smooth curves. The regularity of $H_{w^{(0)}}$ can be obtained by the following lemma.

Lemma 2.1 (Parameterized Sard theorem) Let $V \subset R^{n}, U \subset R^{m}$ be open sets, and $\Phi: V \times$ $U \rightarrow R^{(k)} a C^{r}$ map, where $r>\max \{0, m-k\}$. If $0 \in R^{(k)}$ is a regular value of $\Phi$, then for almost all $a \in V, 0$ is a regular value of $\Phi_{a} \equiv \Phi(a, \cdot)$.

Lemma 2.2 Let $H$ be defined as in (3). In addition, let assumptions $\left(\mathrm{A}_{1}\right)-\left(\mathrm{A}_{5}\right)$ hold, let $u_{i}(x), i=1, \ldots, m$ and $v_{j}(x), j=1, \ldots, l$ be $C^{3}$ functions, and let $\xi_{i}(x), i=1, \ldots, m$ and $\eta_{j}(x)$, $j=1, \ldots, l$ be $C^{2}$ functions. Then, for almost all $w^{(0)} \in X^{0} \times R_{++}^{m} \times R^{l}, 0$ is a regular value of $H_{w^{(0)}}$, and $H_{w^{(0)}}^{-1}(0)$ consists of some smooth curves, among which there exists a smooth curve $\Gamma_{w^{(0)}}$ that starts from $\left(w^{(0)}, 1\right)$. 
Lemma 2.3 Let $H$ be defined as in (3). In addition, let assumptions $\left(\mathrm{A}_{1}\right)-\left(\mathrm{A}_{5}\right)$ hold, let $u_{i}(x), i=1, \ldots, m$ and $v_{j}(x), j=1, \ldots, l$ be $C^{3}$ functions, and let $\xi_{i}(x), i=1, \ldots, m$ and $\eta_{j}(x)$, $j=1, \ldots, l$ be $C^{2}$ functions. Then, for almost all $w^{(0)} \in X^{0} \times R_{++}^{m} \times R^{l}$, the projection of the smooth curve $\Gamma_{w^{(0)}}$ onto the $x$-plane is bounded.

Proof If not, then there exists a sequence of points $\left\{\left(x^{(k)}, y^{(k)}, z^{(k)}, \lambda_{k}\right)\right\}_{k=1}^{\infty}$ such that $\left\|x^{(k)}\right\| \rightarrow$ $\infty$ as $k \rightarrow \infty$.

It is easy to show that the following inequality holds:

$$
\|x-\zeta\|^{2}-\left\|x^{(0)}-\zeta\right\|^{2} \leq 2(x-\zeta)^{T}\left(x-x^{(0)}\right) .
$$

By the homotopy equation (3), we have

$$
\begin{aligned}
& H\left(w^{(k)}, w^{(0)}, \lambda_{k}\right) \\
& =\left(\begin{array}{c}
\left(1-\lambda_{k}\right)\left[\nabla f\left(x^{(k)}\right)+\nabla u\left(x^{(k)}\right) y^{(k)}+\lambda_{k} \xi\left(x^{(k)}\right)\left(y^{(k)}\right)^{2}\right]+\lambda_{k}\left(x^{(k)}-x^{(0)}\right) \\
+\left[\nabla v\left(x^{(k)}\right)+\lambda_{k}\left(\eta\left(x^{(k)}\right)-\nabla v\left(x^{(k)}\right)\right)\right] z^{(k)} \\
v\left(x^{(k)}\right) \\
Y^{(k)} u\left(x^{(k)}\right)-\lambda_{k} Y^{(0)} u\left(x^{(0)}\right)
\end{array}\right) \\
& =0 .
\end{aligned}
$$

Multiplying the first equation in (5) by $\left(x^{(k)}-\zeta\right)^{T}$, we get

$$
\begin{aligned}
& \left(1-\lambda_{k}\right)\left(x^{(k)}-\zeta\right)^{T} \nabla f\left(x^{(k)}\right)+\left(1-\lambda_{k}\right)\left(x^{(k)}-\zeta\right)^{T} \nabla u\left(x^{(k)}\right) y^{(k)} \\
& \quad+\left(1-\lambda_{k}\right) \lambda_{k}\left(x^{(k)}-\zeta\right)^{T} \xi\left(x^{(k)}\right)\left(y^{(k)}\right)^{2}+\left(x^{(k)}-\zeta\right)^{T} \nabla v\left(x^{(k)}\right) z^{(k)} \\
& \quad+\left(x^{(k)}-\zeta\right)^{T} \lambda_{k}\left(\eta\left(x^{(k)}\right)-\nabla v\left(x^{(k)}\right)\right) z^{(k)}+\lambda_{k}\left(x^{(k)}-\zeta\right)^{T}\left(x^{(k)}-x^{(0)}\right)=0,
\end{aligned}
$$

i.e.,

$$
\begin{aligned}
& \lambda_{k}\left(x^{(k)}-\zeta\right)^{T}\left(x^{(k)}-x^{(0)}\right) \\
&=-\left(1-\lambda_{k}\right)\left(x^{(k)}-\zeta\right)^{T} \nabla f\left(x^{(k)}\right)-\left(1-\lambda_{k}\right)\left(x^{(k)}-\zeta\right)^{T} \nabla u\left(x^{(k)}\right) y^{(k)} \\
& \quad-\left(1-\lambda_{k}\right) \lambda_{k}\left(x^{(k)}-\zeta\right)^{T} \xi\left(x^{(k)}\right)\left(y^{(k)}\right)^{2} \\
& \quad-\left(x^{(k)}-\zeta\right)^{T}\left[\nabla v\left(x^{(k)}\right)+\lambda_{k}\left(\eta\left(x^{(k)}\right)-\nabla v\left(x^{(k)}\right)\right)\right] z^{(k)} \\
&=-\left(1-\lambda_{k}\right)\left(x^{(k)}-\zeta\right)^{T} \nabla f\left(x^{(k)}\right)-\left(1-\lambda_{k}\right)\left(x^{(k)}-\zeta\right)^{T} \nabla u\left(x^{(k)}\right) y^{(k)} \\
& \quad-\left(1-\lambda_{k}\right) \lambda_{k}\left(x^{(k)}-\zeta\right)^{T} \xi\left(x^{(k)}\right)\left(y^{(k)}\right)^{2}-\left(1-\lambda_{k}\right)\left(x^{(k)}-\zeta\right)^{T} \nabla v\left(x^{(k)}\right) z^{(k)} \\
&-\lambda_{k}\left(x^{(k)}-\zeta\right)^{T} \eta\left(x^{(k)}\right) z^{(k)} .
\end{aligned}
$$

So

$$
\begin{aligned}
& \lambda_{k}\left(\left\|x^{(k)}-\zeta\right\|^{2}-\left\|x^{(0)}-\zeta\right\|^{2}\right) \\
& \quad \leq 2 \lambda_{k}\left(x^{(k)}-\zeta\right)^{T}\left(x^{(k)}-x^{(0)}\right) \\
& \quad=-2\left(1-\lambda_{k}\right)\left(x^{(k)}-\zeta\right)^{T} \nabla f\left(x^{(k)}\right)-2\left(1-\lambda_{k}\right)\left(x^{(k)}-\zeta\right)^{T} \nabla u\left(x^{(k)}\right) y^{(k)}
\end{aligned}
$$




$$
\begin{aligned}
& -2\left(1-\lambda_{k}\right) \lambda_{k}\left(x^{(k)}-\zeta\right)^{T} \xi\left(x^{(k)}\right)\left(y^{(k)}\right)^{2}-2\left(1-\lambda_{k}\right)\left(x^{(k)}-\zeta\right)^{T} \nabla v\left(x^{(k)}\right) z^{(k)} \\
& -2 \lambda_{k}\left(x^{(k)}-\zeta\right)^{T} \eta\left(x^{(k)}\right) z^{(k)} \\
= & -2\left(1-\lambda_{k}\right)\left(x^{(k)}-\zeta\right)^{T}\left(\nabla f\left(x^{(k)}\right)+\nabla u\left(x^{(k)}\right) y^{(k)}+\nabla v\left(x^{(k)}\right) z^{(k)}\right) \\
& +2\left(1-\lambda_{k}\right) \lambda_{k}\left(\zeta-x^{(k)}\right)^{T} \xi\left(x^{(k)}\right)\left(y^{(k)}\right)^{2}+2 \lambda_{k}\left(\zeta-x^{(k)}\right)^{T} \eta\left(x^{(k)}\right) z^{(k)} \\
\leq & -2\left(1-\lambda_{k}\right)\left(x^{(k)}-\zeta\right)^{T}\left(\nabla f\left(x^{(k)}\right)+\nabla u\left(x^{(k)}\right) y^{(k)}+\nabla v\left(x^{(k)}\right) z^{(k)}\right) \\
& +2\left(1-\lambda_{k}\right) \lambda_{k}\left(u(\zeta)^{T} y^{(k)}-u\left(x^{(k)}\right)^{T} y^{(k)}\right)+2 \lambda_{k}\left(v(\zeta)^{T} z^{(k)}-v\left(x^{(k)}\right)^{T} z^{(k)}\right) .
\end{aligned}
$$

Since $u(\zeta)^{T} \leq 0$ and $y^{(k)} \geq 0$, then $u(\zeta)^{T} y^{(k)} \leq 0$. Besides, by the third equation in (5), we have $u\left(x^{(k)}\right)^{T} y^{(k)}=\lambda_{k} u\left(x^{(0)}\right)^{T} y^{(0)}$, thus the following inequality holds:

$$
\begin{aligned}
\lambda_{k}( & \left.\left\|x^{(k)}-\zeta\right\|^{2}-\left\|x^{(0)}-\zeta\right\|^{2}\right) \\
\leq & -2\left(1-\lambda_{k}\right)\left(x^{(k)}-\zeta\right)^{T}\left(\nabla f\left(x^{(k)}\right)+\nabla u\left(x^{(k)}\right) y^{(k)}+\nabla v\left(x^{(k)}\right) z^{(k)}\right) \\
& -2\left(1-\lambda_{k}\right) \lambda_{k} u\left(x^{(k)}\right)^{T} y^{(k)} \\
= & -2\left(1-\lambda_{k}\right)\left(x^{(k)}-\zeta\right)^{T}\left(\nabla f\left(x^{(k)}\right)+\nabla u\left(x^{(k)}\right) y^{(k)}+\nabla v\left(x^{(k)}\right) z^{(k)}\right) \\
& -2\left(1-\lambda_{k}\right) \lambda_{k}^{2} u\left(x^{(0)}\right)^{T} y^{(0)} .
\end{aligned}
$$

Then by (9), we have

$$
\begin{aligned}
& \left(\zeta-x^{(k)}\right)^{T}\left(\nabla f\left(x^{(k)}\right)+\nabla u\left(x^{(k)}\right) y^{(k)}+\nabla v\left(x^{(k)}\right) z^{(k)}\right) \\
& \quad \geq \frac{\lambda_{k}}{2\left(1-\lambda_{k}\right)}\left(\left\|x^{(k)}-\zeta\right\|^{2}-\left\|x^{(0)}-\zeta\right\|^{2}\right)+\lambda_{k}^{2} u\left(x^{(0)}\right)^{T} y^{(0)}
\end{aligned}
$$

When $\left\|x^{(k)}\right\| \rightarrow \infty$, by (10), we have

$$
\begin{aligned}
& \lim _{k \rightarrow \infty}\left(\zeta-x^{(k)}\right)^{T}\left(\nabla f\left(x^{(k)}\right)+\nabla u\left(x^{(k)}\right) y^{(k)}+\nabla v\left(x^{(k)}\right) z^{(k)}\right) \\
& \quad \geq \lim _{k \rightarrow \infty} \frac{\lambda_{k}}{2\left(1-\lambda_{k}\right)}\left(\left\|x^{(k)}-\zeta\right\|^{2}-\left\|x^{(0)}-\zeta\right\|^{2}\right)+\lambda_{k}^{2} u\left(x^{(0)}\right)^{T} y^{(0)} \geq 0
\end{aligned}
$$

which contradicts assumption $\left(A_{1}\right)$.

Theorem 2.1 Let $H$ be defined as in (3), let $f(x), u_{i}(x), i=1, \ldots, m$ and $v_{j}(x), j=1, \ldots$, l be three times continuously differentiable functions, let assumptions $\left(\mathrm{A}_{1}\right)-\left(\mathrm{A}_{5}\right)$ hold, and let $\xi_{i}(x), i=1, \ldots, m$ and $\eta_{j}(x), j=1, \ldots, l$ be twice times continuously differentiable functions. Then for almost all $w^{(0)} \in X^{0} \times R_{++}^{m} \times R^{l}$, there exists a $C^{1}$ curve $(w(s), \lambda(s))$ of dimension 1 such that

$$
H\left(w(s), w^{(0)}, \lambda(s)\right)=0, \quad(w(0), \lambda(0))=\left(w^{(0)}, 1\right) .
$$

When $\lambda(s) \rightarrow 0, w(s)$ tends to a point $w^{*}=\left(x^{*}, y^{*}, z^{*}\right)$. In particular, the component $x^{*}$ of $w^{*}$ is a $K-K$-T point of problem (1). 
Proof By Lemma 2.2, there must be a $C^{1}$ curve $(w(s), \lambda(s))$ of dimension 1 (denoted by $\left.\Gamma_{w^{(0)}}\right)$ such that

$$
H\left(w(s), w^{(0)}, \lambda(s)\right)=0, \quad(w(0), \lambda(0))=\left(w^{(0)}, 1\right) .
$$

By the classification theorem of one-dimensional smooth manifolds, $\Gamma_{w^{(0)}}$ is diffeomorphic either to a unit circle or to a unit interval. For any $w^{(0)} \in X^{0} \times R_{++}^{m} \times R^{l}$, $\partial H_{w^{(0)}}\left(w^{(0)}, 1\right) / \partial w$ is nonsingular, so $\Gamma_{w^{(0)}}$ cannot be diffeomorphic to a unit circle. That is, $\Gamma_{w^{(0)}}$ is diffeomorphic to a unit interval.

Let $\left(w^{*}, \lambda^{*}\right)$ be a limit point of $\Gamma_{w^{(0)}}$. If $\left(w^{*}, \lambda^{*}\right) \in X^{0} \times R_{++}^{m} \times R^{l} \times(0,1)$, because 0 is a regular value of $H_{w^{(0)}},\left(w^{*}, \lambda^{*}\right) \in H_{w^{(0)}}^{-1}(0)$, and the Jacobian matrix of $H$ at $\left(w^{*}, \lambda^{*}\right)$ is of full row rank, then by the implicit function theorem, $\Gamma_{w^{(0)}}$ can be extended at $\left(w^{*}, \lambda^{*}\right)$. This result contradicts the fact that $\left(w^{*}, \lambda^{*}\right)$ is a limit point of $\Gamma_{w^{(0)}}$.

Let $\left(w^{*}, \lambda^{*}\right)=\left(x^{*}, y^{*}, z^{*}, \lambda^{*}\right)$. Thus, $\left(w^{*}, \lambda^{*}\right) \in \partial\left(X \times R_{+}^{m} \times R^{l} \times(0,1]\right)$ and the following three cases are possible:

(a) $\left(w^{*}, \lambda^{*}\right)=\left(x^{*}, y^{*}, z^{*}, \lambda^{*}\right) \in X \times R_{+}^{m} \times R^{l} \times\{0\}$.

(b) $\left(w^{*}, \lambda^{*}\right)=\left(x^{*}, y^{*}, z^{*}, \lambda^{*}\right) \in X^{0} \times R_{++}^{m} \times R^{l} \times\{1\}$.

(c) $\left(w^{*}, \lambda^{*}\right)=\left(x^{*}, y^{*}, z^{*}, \lambda^{*}\right) \in \partial\left(X \times R_{+}^{m} \times R^{l}\right) \times(0,1]$.

Since the equation $H\left(w, w^{(0)}, 1\right)=0$ has a unique solution $\left(w^{(0)}, 1\right)$ in $X^{0} \times R_{++}^{m} \times R^{l} \times\{1\}$, case (b) is impossible.

By the homotopy equation (3), we have

$$
\begin{aligned}
& \left(1-\lambda_{k}\right)\left[\nabla f\left(x^{(k)}\right)+\nabla u\left(x^{(k)}\right) y^{(k)}+\lambda_{k} \xi\left(x^{(k)}\right)\left(y^{(k)}\right)^{2}\right]+\lambda_{k}\left(x^{(k)}-x^{(0)}\right) \\
& \quad+\left[\nabla v\left(x^{(k)}\right)+\lambda_{k}\left(\eta\left(x^{(k)}\right)-\nabla v\left(x^{(k)}\right)\right)\right] z^{(k)}=0, \\
& v\left(x^{(k)}\right)=0, \\
& Y^{(k)} u\left(x^{(k)}\right)-\lambda_{k} Y^{(0)} u\left(x^{(0)}\right)=0 .
\end{aligned}
$$

Let

$$
I(x)=\left\{i=1, \ldots, m: \lim _{k \rightarrow \infty} y_{i}^{(k)}=+\infty\right\}, \quad J(x)=\left\{j=1, \ldots, l: \lim _{k \rightarrow \infty} z_{j}^{(k)}=+\infty\right\} .
$$

If $J(x) \neq \emptyset$, then

$$
\begin{aligned}
& \left(1-\lambda_{k}\right)\left[\nabla f\left(x^{(k)}\right)+\sum_{i \notin I(x)}\left(\nabla u_{i}\left(x^{(k)}\right) y_{i}^{(k)}+\lambda_{k} \xi_{i}\left(x^{(k)}\right)\left(y_{i}^{(k)}\right)^{2}\right)\right] \\
& +\lambda_{k}\left(x^{(k)}-x^{(0)}\right)+\left[\left(\nabla v\left(x^{(k)}\right)+\lambda_{k}\left(\eta\left(x^{(k)}\right)-\nabla v\left(x^{(k)}\right)\right)\right) z^{(k)}\right. \\
& \left.+\left(1-\lambda_{k}\right) \sum_{i \in I(x)}\left(\nabla u_{i}\left(x^{(k)}\right) y_{i}^{(k)}+\lambda_{k} \xi_{i}\left(x^{(k)}\right)\left(y_{i}^{(k)}\right)^{2}\right)\right]=0 .
\end{aligned}
$$

Because $X$ and $(0,1]$ are bounded, by assumption $\left(\mathrm{A}_{2}\right)$, the third part on the left-hand side of (16) tends to infinity as $k \rightarrow \infty$, but the other two parts are bounded, this is impossible. Hence we conclude that the projection of the smooth curve $\Gamma_{w^{(0)}}$ onto the $z$-plane is also bounded. 
In case (c), first, we prove that $y^{*} \notin \partial R_{+}^{m}$. If $y^{*} \in \partial R_{+}^{m}$, then there exist $i_{0} \in\{1, \ldots, m\}$ and a sequence of points $\left\{\left(w^{(k)}, \lambda_{k}\right)\right\} \subset \Gamma_{w^{(0)}}$ such that $y_{i_{0}}^{(k)} \rightarrow y_{i_{0}}^{*}=0$ as $k \rightarrow+\infty$. From (15), we have

$$
y_{i_{0}}^{(k)} u_{i_{0}}\left(x^{(k)}\right)=\lambda_{k} y_{i_{0}}^{(0)} u_{i_{0}}\left(x^{(0)}\right) .
$$

Because $X$ and $(0,1]$ are bounded, when $k \rightarrow+\infty$, the left-hand side of (17) tends to 0 . At the same time, the right-hand side of (17) tends to $\lambda^{*} y_{i_{0}}^{(0)} u_{i_{0}}\left(x^{(0)}\right)$, which is strictly less than 0 . This fact results in a contradiction.

Then we only need to prove that the remainder of case (c) is impossible. If not, then there exists a sequence of points $\left\{\left(w^{(k)}, \lambda_{k}\right)\right\} \subset \Gamma_{w^{(0)}}$ such that $u_{i}\left(x^{(k)}\right) \rightarrow 0$ for some $i \in\{1, \ldots, m\}$ as $k \rightarrow+\infty$. From (15), we obtain $\left\|y^{(k)}\right\| \rightarrow+\infty$. Because $X$ and $(0,1]$ are bounded, there exists a subsequence of points (denoted also by $\left\{\left(w^{(k)}, \lambda_{k}\right)\right\}$ ) such that $x^{(k)} \rightarrow x^{*},\left\|y^{(k)}\right\| \rightarrow$ $+\infty, z^{(k)} \rightarrow z^{*}$, and $\lambda_{k} \rightarrow \lambda^{*}$ as $k \rightarrow+\infty$.

When $\lambda^{*}>0$, from (15), the active index set is $B\left(x^{*}\right)=I\left(x^{*}\right)$. When $\lambda^{*}=0$, the index set $I\left(x^{*}\right) \subset B\left(x^{*}\right)$.

(1) When $\lambda^{*}=1$, from (16), by the fact that $y_{i}^{(k)}$ is bounded for each $i \notin I\left(x^{*}\right)$, assumptions $\left(\mathrm{A}_{1}\right)-\left(\mathrm{A}_{2}\right)$, we conclude that $\lim _{k \rightarrow+\infty}\left(1-\lambda_{k}\right) y_{i}^{(k)}=0$ and $\lim _{k \rightarrow+\infty}\left(1-\lambda_{k}\right)\left(y_{i}^{(k)}\right)^{2}=y_{i}^{*}$. Therefore, when $k \rightarrow+\infty$, (16) becomes

$$
\eta\left(x^{*}\right) z^{*}+\sum_{i \in I\left(x^{*}\right)} \xi_{i}\left(x^{(k)}\right) y_{i}^{*}+x^{*}=x^{(0)},
$$

which contradicts assumption $\left(\mathrm{A}_{3}\right)$.

(2) When $0<\lambda^{*}<1$, rewrite (16) as

$$
\begin{gathered}
\sum_{i \in I(x)}\left(\nabla u_{i}\left(x^{(k)}\right) y_{i}^{(k)}+\lambda_{k} \xi_{i}\left(x^{(k)}\right)\left(y_{i}^{(k)}\right)^{2}\right)+\frac{\lambda_{k}}{1-\lambda_{k}}\left(x^{(k)}-x^{(0)}\right) \\
=-\nabla f\left(x^{(k)}\right)-\sum_{i \pm I(x)}\left(\nabla u_{i}\left(x^{(k)}\right) y_{i}^{(k)}+\lambda_{k} \xi_{i}\left(x^{(k)}\right)\left(y_{i}^{(k)}\right)^{2}\right) \\
-\frac{1}{1-\lambda_{k}}\left(\nabla v\left(x^{(k)}\right)+\lambda_{k}\left(\eta\left(x^{(k)}\right)-\nabla v\left(x^{(k)}\right)\right)\right) z^{(k)} .
\end{gathered}
$$

Let $y_{I}^{(k)}$ be a vector with components $\left(y_{i}^{(k)}\right)^{2}, i \in I\left(x^{*}\right)$. Then set

$$
\rho_{i}^{(k)}=\frac{\left(y_{i}^{(k)}\right)^{2}}{\left\|y_{I}^{(k)}\right\|}, \quad i \in I\left(x^{*}\right) .
$$

Note that $0 \leq \rho_{i}^{(k)} \leq 1$; then there exists a subsequence of $\left\{\rho_{i}^{(k)}\right\}$, still denoted by $\left\{\rho_{i}^{(k)}\right\}$, such that $\rho_{i}^{(k)} \rightarrow \rho_{i}^{*}$ for each $i \in I\left(x^{*}\right)$ as $k \rightarrow+\infty$. Furthermore, the vector with components $\rho_{i}^{*}$, $i \in I\left(x^{*}\right)$ is denoted by $\rho^{*}$; thus, $\left\|\rho^{*}\right\|=1$. Dividing both sides of (19) by $\left\|y_{I}^{(k)}\right\|$ and letting $k \rightarrow+\infty$, we have

$$
\sum_{i \in I\left(x^{*}\right)} \lambda_{i}^{*} \rho_{i}^{*} \xi_{i}\left(x^{*}\right)=0
$$

which contradicts assumption $\left(\mathrm{A}_{2}\right)$.

(3) When $\lambda^{*}=0$, because the nonempty index set $I\left(x^{*}\right) \subset B\left(x^{*}\right)$, the proof of (3) is similar to that of (2). 
From the discussion above, we conclude that (a) is the only possible case. Hence, the $x$-component of $w^{*}$ is a K-K-T point of $(1)$.

\section{Algorithmic analysis}

For almost all $w^{(0)}=\left(x^{(0)}, y^{(0)}, 0\right) \in X^{0} \times R_{++}^{m} \times R^{l}$, by Theorem 2.1, the homotopy generates a $C^{1}$ curve $\Gamma_{w^{(0)}}$, by differentiating the first equation in (12), we obtain the following theorem.

Theorem 3.1 The homotopy path $\Gamma_{w^{(0)}}$ is determined by the following initial value problem to the ordinary differential equation:

$$
D H_{w^{(0)}}(w(s), \lambda(s))\left(\begin{array}{l}
\dot{w}(s) \\
\dot{\lambda}(s)
\end{array}\right)=0, \quad(w(0), \lambda(0))=\left(w^{(0)}, 1\right),
$$

where $s$ is the arc length of the curve $\Gamma_{w^{(0)}}$.

As for how to trace numerically the homotopy path, there have been many predictorcorrector algorithms, see [20], etc. for reference. Hence we omit them in this paper. In the implementation of the algorithm, generally we need to be devoted to finding the positive direction of the tangent vector at a point on $\Gamma_{w^{(0)}}$ which keeps the sign of the determinant $\left|{ }_{p^{T}}^{\left.D H_{w}()^{(}, \lambda\right)}\right|$ invariant. On the first iteration, the sign is determined by the following lemma.

Lemma 3.1 If $\Gamma_{w^{(0)}}$ is smooth, then the positive direction $p^{(0)}$ at the initial point $\left(w^{(0)}, 1\right)$ satisfies sign $\left|\underset{w^{(0)}{ }^{T}}{D H_{(0)}\left(w^{(0)}, 1\right)}\right|=(-1)^{m+l+1}$.

Proof Let

$$
a=-\nabla f\left(x^{(0)}\right)-\nabla u\left(x^{(0)}\right)+(1-2 \lambda) \xi\left(x^{(0)}\right)\left(y^{(0)}\right)^{2}, \quad b=-Y^{(0)} g\left(x^{(0)}\right),
$$

then

$$
\frac{\partial H_{w^{(0)}\left(w^{(0)}, 1\right)}}{\partial(w, \lambda)}=\left(\begin{array}{cccc}
I & 0 & \eta\left(x^{(0)}\right) & a \\
\nabla v\left(x^{(0)}\right)^{T} & 0 & 0 & 0 \\
Y^{(0)} \nabla u\left(x^{(0)}\right)^{T} & \operatorname{diag}\left(u\left(x^{(0)}\right)\right) & 0 & b
\end{array}\right)=\left(M_{1}, M_{2}\right)
$$

where $M_{1} \in R^{(n+m+l) \times(n+m+l)}, M_{2} \in R^{(n+m+l) \times 1}$. The tangent vector $p^{(0)}$ of $\Gamma_{w^{(0)}}$ at $\left(w^{(0)}, 1\right)$ satisfies

$$
\left(M_{1}, M_{2}\right) p^{(0)}=\left(M_{1}, M_{2}\right)\left(\begin{array}{c}
p_{1}^{(0)} \\
p_{2}^{(0)}
\end{array}\right)=0
$$

where $p_{1}^{(0)} \in R^{n+m+l}, p_{2}^{(0)} \in R^{1}$.

It is easy to get $p_{1}^{(0)}=-M_{1}^{-1} M_{2} p_{2}^{(0)}$, then

$$
\begin{aligned}
\left|\begin{array}{c}
D H_{w^{(0)}\left(w^{(0)}, 1\right)} \\
p^{(0)^{T}}
\end{array}\right| & =\left|\begin{array}{cc}
M_{1} & M_{2} \\
p_{1}^{(0)^{T}} & p_{2}^{(0)^{T}}
\end{array}\right|=\left|\begin{array}{cc}
M_{1} & M_{2} \\
-M_{2}^{T} M_{1}^{-T} & 1
\end{array}\right| p_{2}^{(0)} \\
& =\left|M_{1}\right| p_{2}^{(0)}\left(1+M_{2}^{T} M_{1}^{-T} M_{1}^{-1} M_{2}\right)
\end{aligned}
$$




$$
\begin{aligned}
& =\left|\begin{array}{ccc}
I & 0 & \eta\left(x^{(0)}\right) \\
\nabla v\left(x^{(0)}\right)^{T} & 0 & 0 \\
Y^{(0)} \nabla u\left(x^{(0)}\right)^{T} & \operatorname{diag}\left(u\left(x^{(0)}\right)\right) & 0
\end{array}\right| p_{2}^{(0)}\left(1+M_{2}^{T} M_{1}^{-T} M_{1}^{-1} M_{2}\right) \\
& =\left|\operatorname{diag}\left(u\left(x^{(0)}\right)\right)\right|\left|\begin{array}{cc}
I & \eta\left(x^{(0)}\right) \\
\nabla v\left(x^{(0)}\right)^{T} & 0
\end{array}\right| p_{2}^{(0)}\left(1+M_{2}^{T} M_{1}^{-T} M_{1}^{-1} M_{2}\right) \\
& =(-1)^{l}\left|\operatorname{diag}\left(u\left(x^{(0)}\right)\right)\right|\left|\nabla v\left(x^{(0)}\right)^{T} \eta\left(x^{(0)}\right)\right| p_{2}^{(0)}\left(1+M_{2}^{T} M_{1}^{-T} M_{1}^{-1} M_{2}\right) .
\end{aligned}
$$

Since $u\left(x^{(0)}\right)<0,\left(1+M_{2}^{T} M_{1}^{-T} M_{1}^{-1} M_{2}\right)>0$, and $p_{2}^{(0)}<0$, so the sign of

$$
\left|\begin{array}{c}
D H_{w^{(0)}}\left(w^{(0)}, 1\right) \\
p^{(0)^{T}}
\end{array}\right|
$$

is $(-1)^{m+l+1}$.

The following pseudocode describes the basic steps of a generic predictor-corrector method.

\section{Algorithm 3.1 (Euler-Newton method)}

Step 0 . Provide an initial guess $\left(w^{(0)}, 1\right)$, an initial step length $h_{0}>0$, and three small positive numbers $\epsilon_{1}>0, \epsilon_{2}>0$, and $\epsilon_{3}>0$. Set $k=0$.

Step 1. Compute the direction $\theta^{(k)}$ of the predictor step.

(a) Compute a unit tangent vector $p^{(k)}$.

(b) Determine the direction $\theta^{(k)}$ of the predictor step as follows:

If the sign of the determinant $\left|\begin{array}{c}D H_{w^{(0)}}^{\left(w^{(k)}, \lambda_{k}\right)} \\ p^{(k)^{T}}\end{array}\right|$ is $(-1)^{m+l+1}$, then $\theta^{(k)}=p^{(k)}$.

If the sign of the determinant $\left|\begin{array}{c}D H_{w(0)}\left(w^{(k)}, \lambda_{k}\right) \\ p^{(k)^{T}}\end{array}\right|$ is $(-1)^{m+l}$, then $\theta^{(k)}=-p^{(k)}$.

Step 2. Compute a corrector point $\left(w^{(k+1)}, \lambda_{k+1}\right)$.

$$
\begin{aligned}
& \left(\bar{w}^{(k)}, \bar{\lambda}_{k}\right)=\left(w^{(k)}, \lambda_{k}\right)+h_{k} \theta^{(k)}, \\
& \left(w^{(k+1)}, \lambda_{k+1}\right)=\left(\bar{w}^{(k)}, \bar{\lambda}_{k}\right)-D H_{w^{(0)}}\left(\bar{w}^{(k)}, \bar{\lambda}_{k}\right)^{+} H_{w^{(0)}}\left(\bar{w}^{(k)}, \bar{\lambda}_{k}\right) .
\end{aligned}
$$

If $\left\|H_{w^{(0)}}\left(w^{(k+1)}, \lambda_{k+1}\right)\right\| \leq \epsilon_{1}$, then let $h_{k+1}=\min \left\{h_{0}, 2 h_{k}\right\}$, and go to Step 3 .

If $\left\|H_{w^{(0)}}\left(w^{(k+1)}, \lambda_{k+1}\right)\right\| \in\left(\epsilon_{1}, \epsilon_{2}\right)$, then let $h_{k+1}=h_{k}$, and go to Step 3 .

If $\left\|H_{w^{(0)}}\left(w^{(k+1)}, \lambda_{k+1}\right)\right\| \geq \epsilon_{2}$, then let $h_{k+1}=\max \left\{2^{-25} h_{0},\left(h_{k} / 2\right)\right\}, k=k+1$, and go to Step 2 .

Step 3. If $\lambda_{k+1} \leq \epsilon_{3}$, then stop. Otherwise, $k=k+1$, and go to Step 1 .

\section{Conclusions}

In this paper, we present a set of unboundedness conditions, under which, we are able to solve nonlinear programming problems on a class of unbounded non-convex sets via the combined homotopy interior point algorithm. The main advantage of the algorithm presented in this paper is that it is a globally convergent algorithm whose initial points can be chosen more easily than the locally convergent algorithms. Since nonlinear programming problems have wide applications in engineering, management, economics, and so on, our results may be useful to propose a powerful solution tool for dealing with these nonlinear problems. In future, we devote our efforts to proposing new techniques to solve nonlinear programming problems in a broader class of unbounded non-convex sets. 


\section{Competing interests}

The authors declare that they have no competing interests.

\section{Authors' contributions}

MS carried out the main work and drafted the manuscript. JL participated in completing the proof of Lemma 2.3. ZX participated in completing the proof of Theorem 2.1. All authors read and approved the final manuscript.

\section{Author details}

${ }^{1}$ School of Mathematical Sciences, Luoyang Normal University, Luoyang, 471022, P.R. China. ${ }^{2}$ National Key Laboratory of Science and Technology on Advanced Composites in Special Environments, Harbin Institute of Technology, Harbin, 150080 , P.R. China.

\section{Acknowledgements}

This work was supported by NSFC-Union Science Foundation of Henan (No. U1304103) and Natural Science Foundation of Henan Province (No. 122300410261).

\section{Received: 1 June 2014 Accepted: 14 July 2014 Published: 15 Aug 2014}

\section{References}

1. Kellogg, RB, Li, TY, Yorke, JA: A constructive proof of the Brouwer fixed-point theorem and computational results. SIAM J. Numer. Anal. 13, 473-483 (1976)

2. Smale, S: A convergent process of price adjustment and global Newton method. J. Math. Econ. 3, 1-14 (1976)

3. Chow, SN, Mallet-Paret, J, Yorke, JA: Finding zeros of maps: homotopy methods that are constructive with probability one. Math. Comput. 32, 887-899 (1978)

4. Garcia, CB, Zangwill, WI: An approach to homotopy and degree theory. Math. Oper. Res. 4, 390-405 (1979)

5. Li, Y, Lin, ZH: A constructive proof of the Poincaré-Birkhoff theorem. Trans. Am. Math. Soc. 347, 2111-2126 (1995)

6. Megiddo, N: Pathways to the optimal set in linear programming. In: Megiddo, N (ed.) Progress in Mathematical Programming, Interior Point and Related Methods, pp. 131-158. Springer, New York (1988)

7. Kojima, M, Mizuno, S, Yoshise, A: A primal-dual interior point algorithm for linear programming. In: Megiddo, N (ed.) Interior Point and Related Methods, pp. 29-47. Springer, New York (1988)

8. Kortanek, KO, Potra, F, Ye, Y: On some efficient interior point algorithms for nonlinear convex programming. Linear Algebra Appl. 152, 169-189 (1991)

9. McCormick, GP: The projective SUMT method for convex programming. Math. Oper. Res. 14, 203-223 (1989)

10. Monteiro, RDC, Adler, I: An extension of Karmarkar type algorithm to a class of convex separable programming problems with global linear rate of convergence. Math. Oper. Res. 15, 408-422 (1990)

11. Zhu, JA: A path following algorithm for a class of convex programming problems. ZOR-Methods Models Oper. Res. 36, 359-377 (1992)

12. Feng, GC, Lin, ZH, Yu, B: Existence of interior pathway to the Karush-Kuhn-Tucker point of a nonconvex programming problems. Nonlinear Anal. 32(6), 761-768 (1998)

13. Lin, ZH, Yu, B, Feng, GC: A combined homotopy interior point method for convex nonlinear programming. Appl. Math. Comput. 84, 193-211 (1997)

14. Feng, GC, Yu, B: Combined homotopy interior point method for nonlinear programming problems. In: Fujita, $H_{1}$ Yamaguti, M (eds.) Advances in Numerical Mathematics: Proceedings of the Second Japan-China Seminar on Numerical Mathematics. Lecture Notes in Numerical and Applied Analysis, vol. 14, pp. 9-16. Kinokuniya, Tokyo (1995)

15. $\mathrm{Yu}, \mathrm{B}, \mathrm{Xu}, \mathrm{Q}$, Feng, GC: On the complexity of a combined homotopy interior method for convex programming. J. Comput. Appl. Math. 200(1), 32-46 (2007)

16. Su, ML, Lv, XR: Solving a class of nonlinear programming problems via a homotopy continuation method. Northeast. Math. J. 24(3), 265-274 (2008)

17. Liu, QH, Yu, B, Feng, GC: A combined homotopy interior point method for nonconvex nonlinear programming problems under quasi normal cone conditions. Acta Math. Appl. Sin. 26, 372-377 (2003)

18. Xu, Q, Yu, B, Feng, GC, Dang, CY: Condition for global convergence of a homotopy method for variational inequality problems on unbounded sets. Optim. Methods Softw. 22, 587-599 (2007)

19. Xu, Q, Lin, ZH: The combined homotopy convergence in unbounded set. Acta Math. Appl. Sin. 27, 624-631 (2004)

20. Allgower, EL, Georg, K: Introduction to Numerical Continuation Methods. SIAM, Philadelphia (2003) 